\title{
Native Öle - Rohstoffquelle für Anwendungen in der Kunststoffindustrie
}

\author{
Michael Herzog, Eckhart Kornejew
}

\section{Einleitung}

Natürliche Öle haben in den vergangen Jahren, nicht nur wegen der ansteigenden Preise für Mineralöle, sondern vor allem unter dem Aspekt der Nachhaltigkeit als nachwachsende Rohstoffe zunehmendes Interesse gefunden [1]. Obwohl sie sich in ihrem chemischen Aufbau als Triglyceride grundlegend von den Kohlenwasserstoffen der Mineralöle unterscheiden, können native Öle mit anwendungsspezifisch modifizierten Eigenschaftsprofilen adäquaten Ersatz für mineralische Produkte in werkstofflichen Anwendungen bieten. So liegt der Anteil von Bioschmierstoffen am Gesamtverbrauch von Schmierstoffen und Hydraulikflüssigkeiten in Deutschland bei 3,4 Prozent [2].

Die Weltagrarmärkte für Ölsaaten zeigen eine Dominanz von Sojabohnen (220 Mio. t). Bereits 1998 wuchsen in den USA 40 \% der Sojabohnen und der Baumwolle mit genetisch modifizierten Pflanzen [3]. In den daraus gewonnenen Pflanzenölen ist die über die Nukleinsäuren bestimmbare genetische Herkunft nicht mehr nachweisbar.

In der Europäischen Union nimmt der Anbau von Raps (15 Mio. t) eine führende Stellung ein. Eine lebhafte Nachfrage kommt hier vor allem aus dem Nahrungsmittelund Energiebereich, die stoffliche Nutzung im Bereich der Kunststoffe spielt bislang eine eher untergeordnete Rolle.

Im Non-Food-Bereich wird der größte Anteil heute in Deutschland und auch in den anderen Ländern der Europäischen Union für Kraftstoffe verwendet. Mit der EURichtlinie 2003/30/EG wird das Ziel verfolgt, bis zum Jahr 2010 den Anteil der Biokraftstoffe auf 5,75 \% zu erhöhen [4]. Im Jahr 2005 wurden in Deutschland allein 1,7 Mio. $t$ Biodiesel auf der Basis von Rapsöl erzeugt [5].

Weitere landwirtschaftliche Produkte mit hohem Potential als Rohstoffe für die Industrie sind beispielsweise Stärke, aber auch Fasern. Generell ist z. B. im Bereich der Fasern ein Trend zu Naturstoffen zu verzeichnen, der sich etwa in der Wiederentdeckung von Flachs und Hanf als heimische Naturfasern ausdrückt. Auch Polymere auf Basis natürlicher Rohstoffe finden immer wieder Anwendungen für Produkte aus Cellulose oder Stärke sowie Produkte auf tierischer Basis. Die natürlichen Polymere werden die künstlichen jedoch nicht verdrängen und schon gar nicht vollständig ersetzen können [6].

Produkte aus natürlichen Rohstoffen behaupten sich dann am Markt, wenn sie über gute Qualitäten verfügen, leicht herzustellen und damit preiswert sind. Gelingt es, den Kunden vom Mehrwert derartiger Materialien zu überzeugen, haben diese Produkte beträchtliches Potential am Markt, insbesondere wenn sie über besondere Eigenschaften verfügen. So tragen z. B. viele Verbraucher lieber natürliche Fasern wie Seide (tierische Basis) oder Baumwolle (pflanzliche Basis).

\section{Anwendungen in der Kunststoff- industrie}

Die Nutzung nachwachsender Rohstoffe für technische Anwendungen ist häufig konfrontiert mit dem Problem schwankender Eigenschaften. So sind spezifische Parameter bei den zumeist aus dem landwirtschaftlichen Anbau stammenden Rohstoffen neben einer $z$. T. ausgeprägten Sortenabhängigkeit geprägt von Variabilitäten hinsichtlich der Standorte (Bodenverhältnisse inkl. Düngung), der Anbaukultur und ggf. des Jahrgangs. Mit zuweilen aufwändigen Aufbereitungsprozessen gelingt es jedoch, eine hinreichende Homogenität und Reproduzierbarkeit der Eigenschaften der Produkte zu erreichen und letztendlich eine gleichbleibende und verlässliche Produktqualität zu garantieren und somit aus einem natürlichen Ausgangsstoff ein technisches Produkt zu erzeugen.

Zur Erzielung gewünschter neuer Eigenschaften können chemische Reaktionen an den reaktiven Zentren von nativen Ölen vorgenommen werden, deren Grundstruktur im folgenden dargestellt ist.

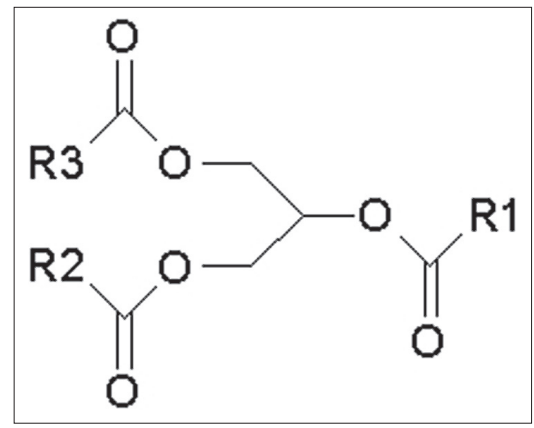

Abb. 1: Chemische Grundstruktur eines nativen Öls als Glyzerinester der Fettsäuren $R 1, R 2$ und $R 3$

Von besonderem Interesse sind neben Umsetzungen an den Esterbindungen (z. B. zu Methylestern) Reaktionen an den Doppelbindungen der ungesättigten Fettsäuren.

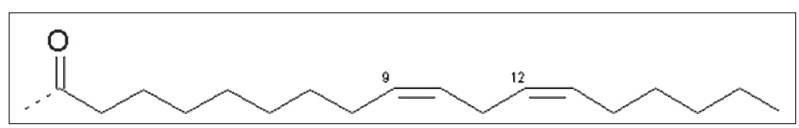

Abb. 2: Chemische Struktur von Linolsäure

Im Rahmen der Forschungs- und Entwicklungsarbeiten der InnoRegio FIRM [7] wurden zwei Projekte bearbeitet, die eine Nutzung nativer Öle für Anwendungen in der Kunststoffindustrie zum Ziel haben.

In den folgenden Tabellen sind die Zusammensetzung und wesentliche chemische Eigenschaften der von uns verwendeten Öle zusammengefasst. 


\begin{tabular}{|l|c|c|c|c|c|c|c|}
\hline Öl & $16: 0$ & $18: 0$ & $18: 1$ & $18: 2$ & $18: 3$ & $\begin{array}{c}12-\mathrm{OH}- \\
18: 1\end{array}$ & $\mathbf{2 2 : 1}$ \\
\hline Leinöl & $4-6$ & $2-4$ & $16-24$ & $14-22$ & $46-56$ & - & - \\
\hline $\begin{array}{l}\text { Rapsöl (eruka- } \\
\text { säurearm) }\end{array}$ & $1-7$ & $1-3$ & $50-70$ & $10-36$ & $1-14$ & - & $0-1$ \\
\hline $\begin{array}{l}\text { Rapsöl (eruka- } \\
\text { säurereich) }\end{array}$ & $1-3$ & $1-12$ & $11-38$ & $1-25$ & $0-10$ & - & $52-64$ \\
\hline Rizinusöl & $1-6$ & $1-8$ & $1-9$ & $3-5$ & $0-1$ & $80-95$ & - \\
\hline Sojäll & $6-11$ & $2-4$ & $20-24$ & $49-55$ & $6-10$ & - & - \\
\hline $\begin{array}{l}\text { Sonnen- } \\
\text { blumenöl }\end{array}$ & $5-8$ & $3-6$ & $20-27$ & $60-68$ & $0-1$ & - & - \\
\hline $\begin{array}{l}\text { Name der } \\
\text { Fettsäure }\end{array}$ & Palmitin- & Stearin- & Öl- & Linol- & Linolen- & Rizinol- & Eruka- \\
\hline
\end{tabular}

Tab. 1: Fettsäurespektrum n:m; Gehalt in \% der Gesamtfettsäuren, Fettsäure: $\mathrm{C}_{n-1} H_{(2 n-2 m-1)} \mathrm{COOH}$ [ $n=$ Länge der Kohlenstoffkette; $m=$ Anzahl der Doppelbindungen]

Hierdurch ist die Anwendungsbreite eingeschränkt, z. B. erfordert die Herstellung von Waschrohstoffen Öle mit 12 und 14 Kohlenstoffatomen, wie sie nur bei Kokos- und Palmkernölen anzutreffen sind, die nicht aus den nördlichen Breiten kommen.

\begin{tabular}{|l|c|c|c|c|c|c|}
\hline Öl & $\begin{array}{c}\rho 20^{\circ} \mathrm{C} \\
\mathbf{g} / \mathrm{cm}^{3}\end{array}$ & $\begin{array}{c}\eta \mathbf{4 0}{ }^{\circ} \mathrm{C} \\
\mathrm{mPas}\end{array}$ & $\begin{array}{c}\mathrm{JZ} \\
\mathbf{g l}_{2} / 100 \mathrm{~g}\end{array}$ & $\begin{array}{c}\mathrm{Fp} . \\
{ }^{\circ} \mathrm{C}\end{array}$ & $\begin{array}{c}\mathrm{M} \\
\mathbf{g} / \mathbf{m o l}\end{array}$ & $\begin{array}{c}\mathrm{M} \\
\text { Säuren } \\
\mathbf{g} / \mathbf{m o l}\end{array}$ \\
\hline Leinöl (roh) & $\begin{array}{c}0,93 \\
\pm 0,006\end{array}$ & 25 & $170-195$ & $-21 \pm 5$ & 860 & $\begin{array}{c}274 \\
279\end{array}$ \\
\hline $\begin{array}{l}\text { Rapsöl } \\
\text { (Vollrafinat) }\end{array}$ & $\begin{array}{c}0,915- \\
0,918\end{array}$ & 40 & $105-120$ & $10 \pm 5$ & 980 & 314 \\
\hline Rapsöl (roh) & $\begin{array}{c}0,910- \\
0,922\end{array}$ & 40 & $95-110$ & - & 881 & 281 \\
\hline Rizinusöl (roh) & $\begin{array}{c}0,96 \\
\pm 0,01\end{array}$ & 232 & $90 \pm 10$ & $-14 \pm 4$ & 923 & 295 \\
\hline $\begin{array}{l}\text { Sojaöl } \\
\text { (Vollrafinat) }\end{array}$ & $\begin{array}{c}0,92 \\
\pm 0,01\end{array}$ & 33 & $130 \pm 10$ & $-13 \pm 3$ & 872 & 278 \\
\hline $\begin{array}{l}\text { Sonnen- } \\
\text { blumenöl (roh) }\end{array}$ & - & 35 & $130 \pm 15$ & $-15 \pm 5$ & 887 & 283 \\
\hline
\end{tabular}

Tab. 2: Charakterisierung der verwendeten Öle anhand der wesentlichen in der Fettchemie verwendeten Parameter

Hierbei stehen $\rho$ für die Dichte, $\eta$ für die Viskosität, JZ für Jodzahl (erfasst den Gehalt an Doppelbindungen), Fp. für den Erstarrungs- bzw. Schmelzpunkt und M für die mittlere Molmasse des Öls bzw. der Fettsäuren des Triglycerids.

Raffinierte Öle sind leicht heller und weisen einen niedrigeren Wasser- und Schmutzgehalt $(<0,1 \%)$ auf, in den physikalischen und chemischen Eigenschaften und in der Zusammensetzung der Fettsäuren sind sie aber nahezu identisch mit den gewöhnlich preisgünstigeren nativen Rohprodukten.

\section{Einführung von Stickstoff zur Erhöhung der Flammfestigkeit}

Unsere Untersuchungen verfolgten das Ziel, neuartige preiswerte und umweltfreundliche Flammschutzmittel für Kunststoffe sowie ein Verfahren zu ihrer Herstellung zu entwickeln. Hierzu wurden native Öle mit hoch stickstoffhaltigen Feststoffen umgesetzt, um somit eine bessere Verteilung der für ihre flammhemmende Wirkung bekannten Stickstoff-Verbindungen in der polymeren Kunststoffmatrix zu erreichen.

Zunächst waren die Reaktionsbedingungen und Trennverfahren zur Herstellung derartiger Flammschutzmittel zu untersuchen. Anschließend wurden durch deren Einsatz flammfest gemachte Kunststoffe bezüglich ihres Brandverhaltens charakterisiert.

Zur Quantifizierung der flammhemmenden Eigenschaften der erhaltenen Reaktionsprodukte in Kunststoffen wurden Basisrezepturen von Polyurethan-Hartschaumstoffen verwendet, wobei das Flammschutzmittel der A-Komponente (Polyol, Katalysator, Treibmittel usw.) des Polyurethansystems beigemischt und anschließend mit der B-Komponente (Poly-Isocyanat) zum Schaumstoff umgesetzt wird.

Zur Beurteilung der Flammfestigkeit wird der Test nach ASTM 1692 herangezogen. Hiernach wird der Grenzsauerstoffkonzentrationsindex LOI (Limiting Oxygen Index) ermittelt. Der LOI bezeichnet die minimale SauerstoffKonzentration in einer Sauerstoff-Stickstoff-Mischung, bei der die Verbrennung eines Probenkörpers gerade noch unterhalten wird. Je höher der LOI ist, desto flammfester ist der getestete Kunststoff. Der LOI wird unter Gleichgewichtsbedingungen einer kerzenartigen Verbrennung in einer nach ASTM D 2863-77 bzw. DIN EN ISO 4589-2 genormten LOI-Kammer bestimmt.

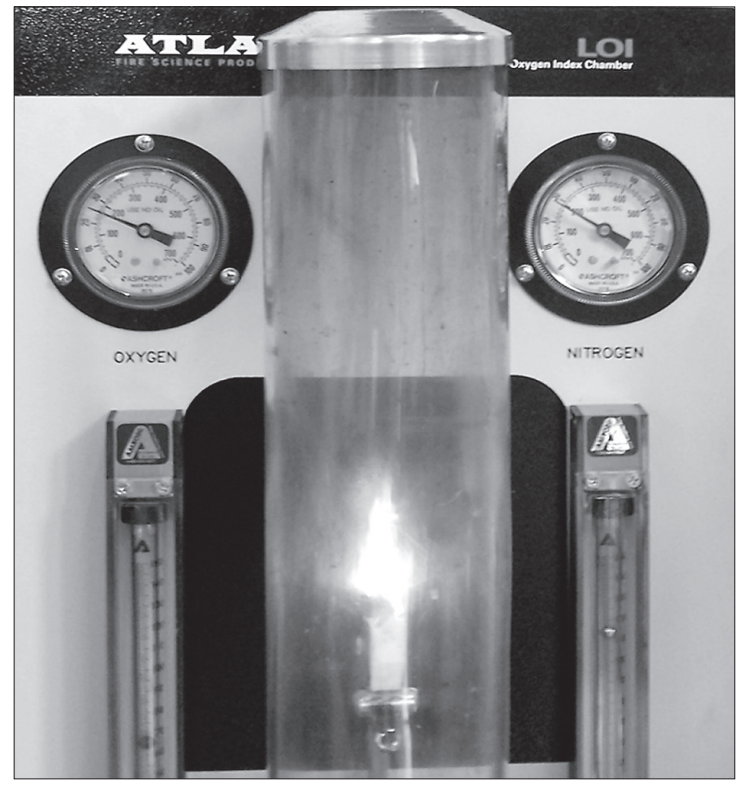

Abb. 3: Bestimmung der Flammfestigkeit mit der LOI-Apparatur

Die Probenkörper der PUR-Hartschaumstoffproben haben Abmessungen von 12,5 x 12,5 x 150 mm. Der LOI wird generell als Durchschnitt aus drei verschiedenen Messungen bei drei unterschiedlichen Gesamtvolumenströmen der Gasmischung mit mehreren Probenkörpern bestimmt. Unter Berücksichtigung von Reproduzierbarkeit und Genauigkeit sind die angegebenen LOI-Werte mit einem Fehler von etwa $\pm 0,05$ behaftet.

Die folgende Tabelle zeigt den LOI für einen StandardPUR-Hartschaumstoff auf Basis Polyesterpolyol $[8,9]$ mit einer variierten Menge Flammschutzkomponente, deren Reaktionsansatz 66,7 \% Melamin enthielt, in Abhängigkeit vom Melaminäquivalent im Polyol. 


\begin{tabular}{|l|c|c|c|l|l|l|}
\hline Melaminäquivalent & \multicolumn{1}{c|}{0} & \multicolumn{1}{c|}{3} & 6 & \multicolumn{1}{c|}{9} & 12 & \multicolumn{1}{c|}{15} \\
\hline LOI & 20,1 & 20,3 & 20,6 & 20,9 & 21,3 & 21,8 \\
\hline Bewertung & $\begin{array}{l}\text { an Luft } \\
\text { bereitwillig } \\
\text { brennbar }\end{array}$ & $\begin{array}{l}\text { an Luft } \\
\text { zögerlich } \\
\text { brennbar }\end{array}$ & $\begin{array}{l}\text { an Luft } \\
\text { zögerlich } \\
\text { brennbar; } \\
\text { später selbst- } \\
\text { verlöschend }\end{array}$ & $\begin{array}{l}\text { an Luft } \\
\text { schwer ent- } \\
\text { flammbar; } \\
\text { selbst- } \\
\text { verlöschend }\end{array}$ \\
\hline
\end{tabular}

Tab. 3: Flammfestigkeit eines PUR-Hartschaumstoffs unter Einsatz Stickstoff-funktionalisierter nativer Öle

Mit diesen Ergebnissen konnte gezeigt werden, dass mit dem von uns verfolgten Ansatz eine flammhemmende Wirkung für Polyurethan-Hartschaumstoffe erreicht werden kann und ein ggf. brandfördernder Effekt durch die Zugabe von Ölen mit deren Stickstoff-Funktionalisierung auf jeden Fall überkompensiert werden kann.

\section{Mehrfach hydroxylierte Fettsäuren für die Polyurethanchemie}

Einen weiteren Untersuchungsgegenstand bildet die Entwicklung von mehrfach hydroxylierten Fettsäuren für Anwendungen als Polyol-Komponente [10] zur Herstellung harter Polyurethane. Eine hohe Hydroxylfunktionalität kann aus natürlichen Pflanzenölen über den Weg einer Epoxidierung an den Doppelbindungen und die damit verbundene Einführung von C-O-Bindungen an der Kohlenstoffkette erreicht werden [11].

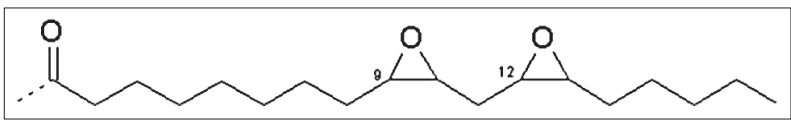

Abb. 4: Chemische Struktur von epoxidierter Linolsäure

In einem weiteren Schritt wurde von uns eine Hydrolyse vorgenommen und ein für die Umsetzung mit Isocyanaten zu Polyurethanen geeignetes Produkt erhalten.

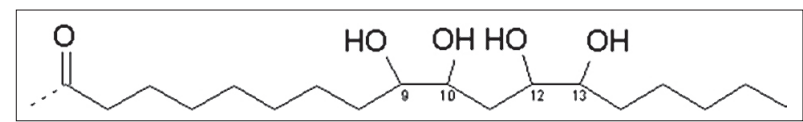

Abb. 5: Chemische Struktur von hydroxylierter Linolsäure

Hierbei ist insbesondere auf den Nachweis der Reaktion an der Doppelbindungen und nicht an den Carboxyl- bzw. Estergruppen zu achten, was uns über IR-spektroskopische Untersuchungen gelang.

Wesentliches Charakteristikum der auf diese Weise hergestellten Polyurethane bzw. PUR-Hartschaumstoffe ist ihre im Vergleich zum eingesetzten Ausgangsöl auffällige Geruchsfreiheit.

Für zukünftige Arbeiten besteht eine weitere in diesem Themenfeld von uns verfolgte Idee darin, durch direkte elektrochemische Hydroxylierung an den Doppelbindungen Polyole aus nativen Ölen herzustellen. Damit wird die Entwicklung eines technisch realisierbaren Verfahrens mittels neuartiger, mit Nanaostrukturen beschichteten Elektroden angestrebt, die eine hohe Konzentration von Hydroxylradikalen in wässriger Lösung oder Dispersion erzeugen.

\section{Literatur/Anmerkungen}

[1] P. Hövelmann: Nachwachsende Rohstoffe für die Chemie, S. 21 Landwirtschaftsverlag Münster, 2001

[2] Situationsbericht 2006, Trends und Fakten zur Landwirtschaft, Deutscher Bauernverband 2005

[3] L. Willmitzer: 6th Symposium on Renewable Resources, S. 29, Landwirtschaftsverlag Münster, 1999

[4] Richtlinie 2003/30/EG des Europäischen Parlaments und des Rates vom 8. Mai 2003 zur Förderung der Verwendung von Biokraftstoffen oder anderen erneuerbaren Kraftstoffen im Verkehrssektor

[5] Bundesministerium für Umwelt, Naturschutz und Reaktorsicherheit: Entwicklung der Erneuerbaren Energien 2005, Aktueller Sachstand März 2006

[6] Fraunhofer-Institut für Angewandte Polymerforschung IAP, Jahresbericht 2005/2005

[7] M. Herzog, K. Erxleben: Wiss. Beitr. Techn. Fachhochschule Wildau 2005, S. 8

[8] R. Langenstraßen, S. Fulev, A. Apel, B. Gebert, D. Lehmann, G. Behrendt: Entwicklung der Grundlagen für eine Laboranlage zur kontinuierlichen Herstellung von PET-Recyclingpolyolen, Wiss. Beitr. Techn. Fachhochschule Wildau 2004, S. 34

[9] G. Behrendt, M. Pohl: Polyester-Polymerpolyole für Polyurethane und Verfahren zu ihrer Herstellung, DE 19915125 C2 (2001)

[10] G. Adolf, U. Erkens: Bindemittel und Additive für hochwertige Polyurethanbeschichtungen, Cognis Deutschland GmbH, 2004

[11] Scientific Panel AFC: On a request from the Commission related to the use of Epoxidised soybean oil in food contact materials, The EFSA Journal (2004)64, 1-17

\section{Autoren}

Dr. rer. nat. Michael Herzog

Technische Fachhochschule Wildau

Fachbereich Ingenieurwesen/Wirtschaftsingenieurwesen Geschäftsstelle InnoRegio FIRM

Tel. + 493375 508-332

michael.herzog@igw.tfh-wildau.de

Dipl.-Chem. Eckhart Kornejew

Technische Fachhochschule Wildau

Fachbereich Ingenieurwesen/Wirtschaftsingenieurwesen

Tel. + 493375 508-384

ekorneje@igw.tfh-wildau.de 